\title{
THE CHANGING SPECTRUM AND ORBITAL MOTION OF THE SMC WOLF-RAYET BINARY HD 5980
}

\author{
R.H. BARBÁ ${ }^{1 *}$ and V.S. NIEMELA ${ }^{2 * *}$ \\ ${ }^{1}$ Observatorio Astronómico, Universidad Nacional de La Plata, Argentina \\ ${ }^{2}$ Instituto de Astronomía y Física del Espacio, Buenos Aires, Argentina
}

\begin{abstract}
Optical spectroscopic observations of the eclipsing binary WR star HD 5980 in the SMC, obtained during different epochs, show a remarkable change of the WR type spectrum from spectral type WN3-4 to WN7-8. Radial velocities of the WR emission lines from spectra obtained in August 1992, indicate an orbital motion corresponding to the secondary component which is in front of the system during the primary eclipse. We compare the RV curves and phase-dependent line-width variations observed in 1992 with previously published data.
\end{abstract}

Key words: stars: Wolf-Rayet - binaries - variables - individual: HD 5980

\section{Introduction and observations}

The brightest WR object in the SMC, the eclipsing binary system HD 5980 $\left(P=19^{\mathrm{d}} .266\right.$, Breysacher \& Perrier 1980), has been classified differently in time, e.g., OB+WN3 (Walborn 1977); WN4+O7I: (Breysacher et al. 1982); and WN4.5+WN3 + a line-of-sight OI companion based on the RV behavior and intensity ratios of NIV $4058 \AA$ and NV $4603 \AA$ emission, and H9 and HeI $4471 \AA$ absorption lines (Niemela 1988).

Here, we present a spectral study of radial velocities and the other parameters of emission lines of HD 5980, observed over an almost complete orbital cycle during August 1992. We also describe the extraordinary changes in the optical spectrum of HD 5980 over the last 14 years. We have obtained digital spectra at CASLEO and CTIO during 1985-1994. Photographic material was secured at CTIO during 1980-1983 (cf. Niemela 1988).

\section{Results and discussion}

Our 1992 data for the HeII $4541 \AA$ NIII $4640 \AA$ and NV $4603 \AA$ emission lines, show for each of them a similar RV distribution, suggesting that these lines are formed in the same source, i.e., in one WN component which is in front of the system during the primary eclipse (phase 0.0). In contrast, the HeII $4686 \AA$ emission line displays a complicated RV behavior indicating, perhaps, contributions from two different WN stars. We note that the FWHM-curve of the HeII $4686 \AA$ emission line over the orbital cycle in August 1992 resembles the one published by Breysacher et al. (1982). On the other hand, the

* Fellow of CONICET and Visiting Astronomer CASLEO, San Juan, Argentina

** Member of Carrera del Invetigador Científico, CIC, Prov. Bs.As., Argentina and Visiting Astronomer CTIO, NOAO operated by AURA 


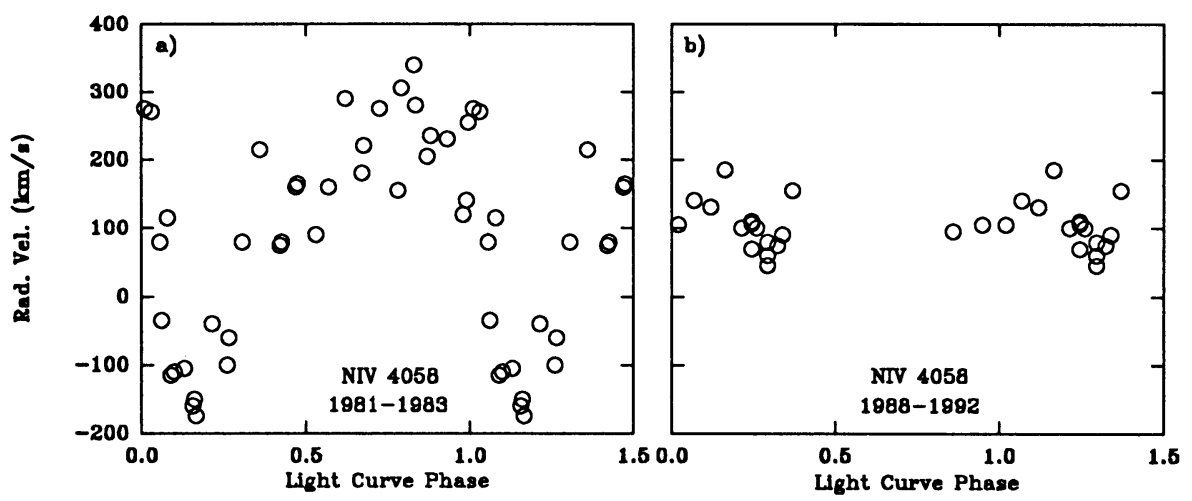

Fig. 1. HD 5980: RV of the NIV $4058 \AA$ emission line folded with $P=19^{\mathrm{d}} .266$. Left pannel: data obtained between 1980-1983. Right pannel: data obtained between 1988-1992.

NIV $4058 \AA$ emission line, which in the 1981-1983 data showed a large amplitude RV curve corresponding to the component which is behind during the primary eclipse (Niemela 1988), does not show appreciable RV variations in our data from 1988-1992 (Fig. 1). Summarizing the spectacular changes of the emission lines of HD 5980 in our data of the last 14 years:

1980-'83: Nve > Nive, NiIle weak or absent, Hi absorption present.

1985.96: Nive > Nve, NiIle absent, Hele weak.

1988.08: Nive $>$ Nille $>$ Nve, Hele weak.

1992.67: Nive strong $>$ Nve, Nive $\sim$ NiIle, Hele weak.

1993.92: HeI P-Cyg strong, Nille strong, Nive weak, Nve absent.

1994.08: HeI P-Cyg, Ninle > Nive, Nve weak.

We conclude from the observed spectral changes that the HD 5980 binary system has suffered an outburst-like event, as also reported for the UV region by Koenigsberger et al. (1994). Both binary components in HD 5980 exhibit large spectral variations, which render their spectral classification ambiguous. Obviously, more observations of HD 5980 are warranted.

\section{Acknowledgements}

We thank P. Bartzakos for the loan of two spectra, and M. Shara and D. Golombek for hospitality at STScI, where a first version of this paper was written.

\section{References}

Breysacher, J., Moffat, A.F., Niemela V.S. 1982, ApJ 257, 116

Breysacher, J., Perrier, C. 1980, $A \& A$ 90, 207

Koenigsberger, G. et al. 1994 preprint

Niemela, V.S. 1988, in: V.M. Blanco \& M.M. Philips (eds.), Progress and Opportunities in Southern Hemisphere Optical Astronomy, ASP Conf. Series 1, 381

Walborn, N.R. 1977, ApJ 215, 53 